\title{
Percutaneous Ultrasound-Guided Radiofrequency Thermal Ablation for Treatment of Uterine Fibroids
}

\author{
Raúl García Marcos ${ }^{1 *}$, Javier Monleón², Alicia Martínez-Varea², Fernando Gómez ${ }^{3}$, \\ Guillermina Montoliú1, Jose J. Martínez¹, Luis Martí-Bonmatí1, Antonio Pellicer² \\ ${ }^{1}$ Department of Radiology, La Fe University Hospital, Valencia, Spain \\ ${ }^{2}$ Department Obstetrics and Gynecology, La Fe University Hospital, Valencia, Spain \\ ${ }^{3}$ Department of Radiology, Hospital Clinic, Barcelona, Spain \\ Email: ${ }^{*}$ raulgamar@gmail.com
}

Received 20 June 2014; revised 15 July 2014; accepted 13 August 2014

Copyright @ 2014 by authors and Scientific Research Publishing Inc.

This work is licensed under the Creative Commons Attribution International License (CC BY).

http://creativecommons.org/licenses/by/4.0/

(c) (i) Open Access

\section{Abstract}

Purpose: To assess the safety, efficacy and effectiveness of percutaneous radiofrequency (RF) thermal ablation to reduce symptoms of uterine fibroids. Materials and Methods: 17 premenopausal women with symptomatic uterine fibroids despite conventional medical treatment were included. The assessment of symptoms and characteristics of fibroids by vaginal ultrasound, magnetic resonance imaging (MRI) and contrast enhanced ultrasound (CEUS) was performed before starting treatment and 6 months after the procedure. Successful treatment was clinically considered if patients reported a reduction in symptoms 6 months after RF myolysis. Successful treatment was also considered if the necrosis of the fibroid was greater than $50 \%, 6$ months after treatment. Results: The baseline score on visual analogue scale (VAS) for dysmenorrhea and intermenstrual pain was $5.76 \pm 3.31$ and $3.0 \pm 3.4$, respectively. According to a score of $0-3$, baseline bleeding during menstruation was $2.29 \pm 0.92$. Six months after RF myolysis, the VAS for dysmenorrhea was $2.75 \pm 3.32(p=0.004)$, whereas for intermenstrual pain it was $1.38 \pm 2.56(p=0.02)$. Menstrual bleeding was reduced to $1.13 \pm 0.89(p=0.005)$. Clinical success of the treatment was evident in $11(64.7 \%)$ of the 17 patients with a 95\% CI [38.6\%, 84.7\%]. Fourteen patients underwent MRI monitoring 6 months post-myolysis. Compared to baseline fibroid volume, ultrasound and MRI volume were $57.38 \%$ and $79.66 \%$ six months after surgery, respectively. A total of 13 patients $(92.86 \%)$ had radiological success from the treatment (95\% CI [64.2\%, 99.6\%]). Conclusion: Since percutaneous RF myolysis reduces volume and symptoms of uterine fibroids, it may be considered as a valid treatment for symptomatic fibroids.

\footnotetext{
${ }^{*}$ Corresponding author.
} 


\section{Keywords}

\section{Percutaneous Radiofrequency Thermal Ablation, Myolysis, Uterine Fibroids}

\section{Introduction}

Uterine fibroids are the most common tumourin women [1]-[3]. Actually, up to $80 \%$ women will have at least one leiomyoma [2]. These monoclonal tumours arise from the uterine smooth-muscle tissue and depend on oestrogen and progesterone [2]. A single genetic hit might lead clonal expansion of stem cells within the normal myometrium [2].

Most uterine fibroids are asymptomatic [1]. Nevertheless, up to half women with symptomatic fibroids present significant clinical symptoms, including excessive uterine bleeding, dysmenorrhoea, intermenstrual pain, pelvic discomfort, infertility and consequent reduction in quality of life [1]-[5].

Diagnosis is generally performed by ultrasound, being deemed transvaginal exploration an excellent procedure due to its precision and availability [1] [6] except large fundal fibroids. MRI is particularly useful to define location of fibroids, being highly precise in problematic cases, such as large ( $>375 \mathrm{~mL})$, fundal or multiple $(>4)$ fibroids [7].

Surgical treatment is the classical management of symptomatic fibroids. Whereas myomectomy is performed in women wishing to preserve their fertility, hysterectomyis carried out in the remaining patients [3] [6].

With technological development, interventional techniques, which allow selective destruction of fibroid tissue in a relatively bloodless manner, have also emerged [1] [8]. These include selective endovascular embolization, high intesity focused ultrasound (HIFU) and RF [4]-[6] [8]. Uterine artery embolization has little selectivity associated with the target [3] [9]. It is not counselled to women with reproductive desire [3] [4] [9]. In addition, the possibility of a fibroid recurrence is not excluded, and $20 \%$ of patients require a subsequent hysterectomy [4]. HIFU makes it possible to necrose the tumour by applying multiple ultrasonic foci concentrated on the fibroid.

RF myolysis is currently an interesting option of treatment due to the development of new electrodes that allow percutaneous or transvaginal use instead of laparoscopic approach [1] [4] [10]. Energy is accurately released through them within the fibroid without affecting surrounding tissues. With a mean treatment time of 20 minutes and an affordable cost, RF has excellent associated postoperative tolerance, thereby allowing reduction in fibroid size and symptoms [4]-[6] [8] [9]. It has been demonstrated that ultrasound-guided transvaginal RF myolysis is a safe, effective and minimally invasive outpatient procedure leading to size reduction and symptoms release [1] [4] [5] [8] [9].

As the vaginal access to afibroid may be difficult due to its location, the application of RF thermal ablation via percutaneous puncture in the treatment of symptomatic uterine fibroids is in favour. This study assessed the technique's safety and efficacy in reducing symptoms. Furthermore, tumour response was evaluated by ultrasound and MRI, which enable objective assessment of the technique's effectiveness.

\section{Material and Methods}

\subsection{Patients}

Inclusion criteria for this prospective study were: 1 ) patients older than 18 years of age with symptomatic uterine fibroids not controlled with medical treatment based on intrauterine devices (IUD) with levonorgestrel (LNG) and/or antifibrinolytics, 2) capable of understanding the risks and benefits of radiofrequency thermocoagulation and its advantages and disadvantages compared with surgery, 3) without further comorbidities and without contraindication to sedation or loco-regional anaesthesia, 4) No response to pharmacological treatment. Exclusion criteria included: 1) women younger than 18 years of age, 2) presence of serious associated pathology, 3) evidence of active infection, 4) coagulation abnormalities, 5) pregnancy or lactation, 6) not voluntarily signing the informed consent, 7) submucosal fibroids 8) reproductive desire. Minimal size was $3.5 \mathrm{~cm}$ and no number of fibroids was considered as exclusion criteria. In this case we treated the biggest.

Between November 2011 and December 2012, 17 premenopausal women were finally selected in the study that was carried out in University \& Polytechnic Hospital La Fe, Valencia (Spain). They had symptomatic uter- 
ine fibroids with persistent symptoms despite conventional medical treatment and refused myomectomy or hysterectomy. All of them underwent percutaneous RF myolysis. All patients were adequately informed of the potential risks and expected benefits of the percutaneous procedure as well as of the possible alternative treatments. Ethics Committee of our hospital approved the study. All patients provided written informed consent.

\subsection{Explorations Prior to the Intervention}

All the patients were assessed by the Gynaecology as well as by the Radiology Department with the following protocol.

The Gynaecology Department conducted 1) a quantification of the dysmenorrhea and intermenstrual pain on a VAS with a score from 0 to 10 (0 represented no pain and 10 was maximum pain), and a quantification of menstrual bleeding with a score from 0 to 3 ( 0 amenorrhea, 1 mild bleeding, 2 moderate and 3 severe), 2) preoperative analytical assessment of baseline haematocrit, and 3) transvaginal ultrasound (Acuson S2000 ultrasound system, Siemens, Erlangen, Germany; EV-8C4 Transducer, 4 - $9 \mathrm{MHz}$ ) to define the number, location (fundal, anterior, posterior, right side, left side, right horn, left horn), situation (subserosal, intramural or submucosal) and size (maximum diameter and volume according to the formula $\mathrm{V}=0.5233 \times \mathrm{D} 1 \times \mathrm{D} 2 \times \mathrm{D} 3$ [11], D1 being anteroposterior, D2 longitudinal and D3 transversal) of fibroids.

The Radiology Department conducted, prior to and after the interventional procedure, 4) an abdominal ultrasound (Acuson S2000 ultrasound system, Siemens, Erlangen, Germany; 4C1 Transducer, 1 - 4.5 MHz) in order to plan percutaneous accessibility and CEUS after administration of $2 \mathrm{~mL}$ of IV contrast agent SonoVue (BraccoSpA, Milan, Italy) followed by $10 \mathrm{~mL}$ of physiologic saline solution (PSS) by bolus in order to assess fibroid perfusion (enhancement and percentage of necrosis). Also, 5) a Magnetic Resonance Imaging (MRI) was carried out using either a $1.5 \mathrm{~T}$ or a $3 \mathrm{~T}$ equipment (SignaHDxt $1.5 \mathrm{~T}$ and SignaHDxt 3.0 T, General Electric, Wauwatosa, Wisconsin, United States) with the following sequences. Single shot T2 weighted fast spin echo in the coronal plane (matrix: $384 \times 224$, FOV: $440 \times 440$, slice thickness: $4 \mathrm{~mm}$ ). Single-shot spin echo T2 weighted sequences in the transverse and coronal planes (matrix: $384 \times 224$, FOV: according to patient's constitution, slice thickness: $5 \mathrm{~mm}, 1 \mathrm{~mm}$ apart). Diffusion weighted imaging in the transverse plane (matrix: $132 \times 132$, FOV: depending on patient, slice thickness: $5 \mathrm{~mm}, 1 \mathrm{~mm}$ apart, 2 b values 0 and 800). Finally, a 3D T1weighted gradient echo with fat-suppression (LAVA) was obtained in the coronal plane (matrix: $320 \times 224$, FOV: $440 \times 440$, slice thickness: $2 \mathrm{~mm}$, without separation) obtaining 5 phases (precontrast, 30, 70, 120 and 420 seconds) after the administration of $0.2 \mathrm{ml} / \mathrm{kg}$ body weight of intravenous contrast agent Multihance ${ }^{\circledR}$ (Bracco, Singen, Germany). Fibroid volume was assessed by MRI $(\mathrm{V}=0.5233 \times \mathrm{D} 1 \times \mathrm{D} 2 \times \mathrm{D} 3)$ [11] as were the contrast enhancement, signal intensity on T2-weighted sequences, tumour uniformity compared to the myometrium, and percentage of necrosis compared to baseline fibroid $(<50 \%, 50 \%-75 \%$ or $>75 \%)$.

\subsection{Interventional Technique}

The RF myolysis procedure was performed in the interventional radiology surgery room under intravenous sedation with 1 - 3 mg of midazolam (B Braun Medical S.A., Spain), 25 - 50 mcg i.v. fentanyl (Prostrakan Limited, S.A., Galashiels, United Kingdom) and 50 - $120 \mathrm{mg}$ of propofol (PropofolLipuro ${ }^{\circledR}$, B Braun Medical S.A., Barcelona, Spain). Patients were placed in dorsal lithotomy position and monitored by means of electrocardiogram, blood pressure and pulse oximeter.

Broad-spectrum antibiotic prophylaxis based on amoxicillin + clavulanic acid (Augmentin; Glaxo-Smithkline Beecham, Brentford, United. Kingdom) and urethral catheterization were done at the beginning of the procedure. A cohen uterine manipulator (QuickMedical, Issaquah, Washington) was applied to allow fixation only in those patients with difficulty to puncture the fibroiddue to its rigidity or location (in the upper third).

Next, the fibroid was punctured under abdominal ultrasound monitoring. In all cases, prior to thermal ablation, a biopsy was performed using an $18 \mathrm{G}$ BioPince ${ }^{\circledR}$ needle (Angiotech, Vancouver, British Columbia, Canada) inserted through the RF coaccess needle to use the same puncture and determine its pathology. RF was performed using a coaxial system with a 35 to $40 \mathrm{~mm}$ long umbrella-shaped needle-electrode (LeVeenCoAccess, RF3000 system, Boston, USA). One or more thermal ablations were performed depending on the size of the fibroid. CEUS was then repeated with $2 \mathrm{~mL}$ of IV contrast agent and $10 \mathrm{~mL}$ of PSS to confirm the extent of necrosis and assess needle replacement (Figure 1). Ablation of the tract with $10 \mathrm{~W}$ of power was carried out at the end of the procedure in order to prevent bleeding. 


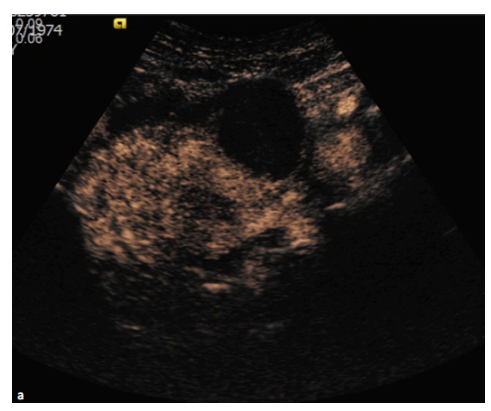

(a)

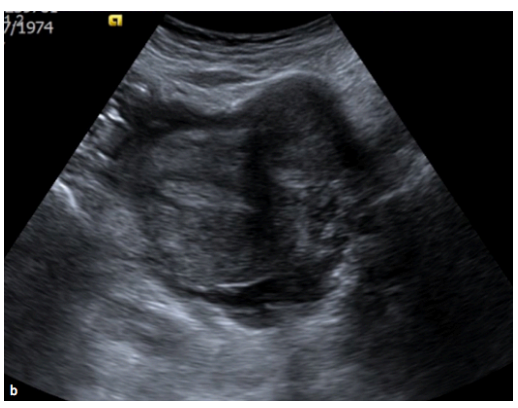

(b)

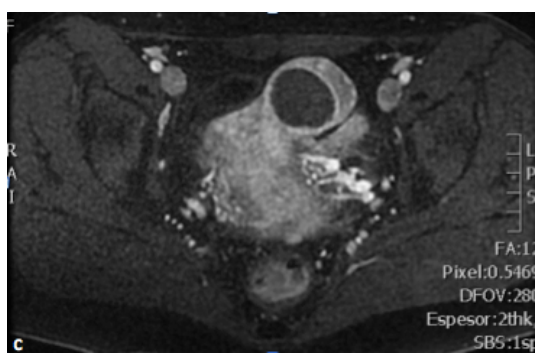

(c)

Figure 1. Intramural fibroid treated by RF. CEUS in the immediate postoperative period (a); abdominal ultrasound (b); transverse MRI image of fat-suppressed T1-weighted sequence and contrast 6 months after treatment (c).

Surgical time, the maximum intensity applied by the RF and number of ablations performed were recorded. CEUS was used to determine the appearance of the perfusion and percentage of necrosis of the fibroid treated $(<50 \%, 50 \%-75 \%$ or $>75 \%)$ prior to and 6 months post-treatment.

\subsection{Data Collection}

Assessment of symptoms and fibroids characteristics by vaginal ultrasound, MRI and CEUS were performed both before starting treatment (baseline) and 6 months after RF myolysis. A single gynaecologist and radiologist with more of 4 years of experience performed both the vaginal gynaecological ultrasound evaluation and the evaluation of MRI and CEUS to reduce interobserver variations. When several fibroids were found in a single patient, only the largest one was treated and assessed.

The incidence of major and minor intra- and post-operative complications was collected on all the patients. Minor complications were considered as temporary and self-limiting symptoms requiring no treatment or medical treatment for a period of less than ten days, and did not generate any sequelae. Major complications were defined as those that required further intervention and/or hospitalisation.

Successful treatment was clinically considered when observing a reduction in symptoms 6 months after RF myolysis. Successful treatment was also considered when the achieved fibroid necrosis at 6 months after treatment was larger than $50 \%$.

\subsection{Statistical Analysis}

Continuous data are presented as mean (standard deviation) and categorical data are expressed in relative and absolute frequencies. Differences between pre and post RF myolysis were assessed by paired Wilcoxon tests. The Statistical Package for the Social Sciences, version 12.0 (SPSS Inc., Chicago, IL, USA) was used for the statistical analysis.

\section{Results}

Mean age of the 17 included patients was 44.0 (5.1) years. The majority were nulligravida (13; 76.50\%). The median number of gestations of the 4 remaining women was 2 . Regarding the lesion, 10 patients $(58.82 \%)$ had a single fibroid and 7 (41.18\%) had 2 or more fibroids. The location of the fibroids was in 5 cases (29.4\%) anterior, in $2(11.7 \%)$ cases posterior, in 6 cases (35.3\%) fundic, in 2 cases (11.7\%) in right side and in 2 cases (11.7\%) in the right horn. Regarding the type of fibroids, 5 (29.4\%) were subserosal and 12 (70.5\%) intramural.

The mean operation time was 35.9 (10.6) min (range 30 - $55 \mathrm{~min}$ ). It was necessary to apply a cohen uterine manipulator in 6 cases (35.2\%). A maximum intensity of 116.18 (34.17) W was used. The number of ablations performed in each patient to cover the widest possible volume of fibroid was 2.3 (1.0) (range 1 - 5) with one puncture and assess needle replacement.

All patients were discharged within the first 24 hours after the intervention. Mean time in hospital was 12 hours (range 8 - 24).

No intraoperative complications were observed. Postoperative complications associated with the procedure were observed in 5 patients (29.4\%) in the first 15 days. None of them reported serious complications. Three 
patients presented mild postoperative pain, which subsided with anti-inflammatory drugs in fewer than 7 days following the procedure. One patient developed cutaneous erythema at the puncture site, which disappeared over the first 10 days after myolysis with a topical treatment of nitrofural based ointment. Severity of these complications was deemed mild. One patient presented a burn of first degree in the thigh, which subsided with nitrofural ointment during the first month after surgery. The severity of this complication was deemed to be moderate.

Regarding late complications after myolysis, 1 patient (6.2\%) underwent laparoscopic hysterectomy one month after the procedure due to the appearance of the fibroid through the external cervical os. Subsequent revision of the case revealed that the fibroid (maximum diameter $65 \mathrm{~mm}$ ), despite being largely intramuscular, displayed a significant submucosal component with risk of detachment.

The anatomopathological study confirmed presence of fibroid tissue in all lesions ablated by percutaneous radiofrequency.

A total of 16 of the 17 (94.1\%) patients completed clinical follow-up 6 months post-myolysis. One patient did not complete the study because she required a hysterectomy. Table 1 shows the VAS outcomes before and 6 months after myolysis. The baseline dysmenorrhea and intermenstrual pain was 5.76 (3.31) and 3.0 (3.4) respectively. According to a score of 0 - 3, baseline bleeding during menstruation was 2.29 (0.92). Interestingly, 14 women $(82.4 \%)$ had moderate or severe bleeding (score $\geq 2$ ). Six months after RF myolysis, the VAS for dysmenorrhea was 2.75 (3.32), whereas for intermenstrual pain it was 1.38 (2.56). Menstrual bleeding was reduced to $1.13(0.89)$. Only $5(31.2 \%)$ of the 16 women reported having dysmenorrhea $\geq 5$ and $3(18.7 \%)$ intermenstrual pain $\geq 5$. Only three patients $(18.7 \%)$ had a score $\geq 2$ for menstrual bleeding.

Clinical success of the treatment was evident in 11 (64.7\%) of the 17 patientswith a 95\% CI [38.6\%, 84.7\%]. Only in 6 patients (35.2\%) the procedure did not reduce symptoms in the first 6 months post-myolysis.

The maximum diameter of the fibroid treated by vaginal ultrasound was 62.88 (15.54) mm. Volume by ultrasound and MRI was 112.26 (65.24) cc and 118.71 (74.88) cc, respectively (Table 2). The appearance of the MRI fibroid enhancement was homogeneous in 15 cases (88.2\%) while heterogeneous in 2 (11.7\%) (Table 3). The signal on T2-weighted images was hypointense and homogeneous in 4 cases (23.5\%), hypointense and heterogeneous in 9 cases (52.9\%), hyperintense and homogeneous in 1 case (5.8\%), and hyperintense and heterogeneous in 3 cases (17.6\%). Regarding the DW images, in all cases (100\%) there was no evidence of increased signal intensity on the DW images, the ADC map had no signal intensity changes. Finally, baseline CEUS appearance was, in all cases, hypervascular (100\%) compared to myometrium.

Fourteen of the 17 patients underwent MRI monitoring 6 months post-myolysis. One patient required a prior hysterectomy and two patients did not consent to the MRI monitoring scan. The maximum ultrasound diameter of the fibroid treated 6 months after RF myolysis was 52.00 (16.41) $\mathrm{mm}$. The ultrasound and MRI volume were 64.42 (51.78) and 94.57 (75.32) cc, respectively (Table 2). Compared to baseline fibroid volume, ultrasound and MRI volumes were $57.38 \%$ and $79.66 \%$ six months after surgery, respectively. MRI capture appearance of fibroid 6 months after RF myolysis was in 10 cases (71.4\%) homogeneous and in 4 cases (28.5\%) heterogeneous (Table 3). Regarding fibroid necrosis seen at 6 months post-treatment, 1 case $(7.1 \%)$ presented fibroidnecrosis < $50 \%$, 3 cases (21.4\%) showed necrosis of $50 \%$ to $75 \%$, and 10 cases $(71.4 \%)$ showed fibroid necrosis $>75 \%$. A total of 13 patients $(92.86 \%)$ had radiological success from the treatment, considered as fibroid necrosis $>50 \%$ six months after treatment. Regarding the T2-weighted signal six months after myolysis, in 4 cases (28.5\%) it was heterogeneous hypointense, in 1 (7.1\%) it was homogeneous hyperintense, and in 9 cases (64.2\%) it was heterogeneous hyperintense (Figure 2). Moreover, in all the 14 cases (100\%) there was no MRI evidence of restricted diffusion. CEUS appearance 6 months after treatment in the 15 patients studied was in 14 cases (93\%) avascular, and in 1 case (7\%) hypovascular (Figure 3). Two patients did not complete the CEUS six months post-intervention. These changes indicate the extent and effect of thermal ablation, thereby allowing early prediction of treatment success.

Six months after surgery, 13 (81.2\%) of the 16 patients who completed follow-up did not require further treatments to alleviate the symptoms caused by fibroids. Only three women (18.7\%) needed additional treatment. Persistent symptoms were resolved with symptomatic treatment based on oral gestagens in 2 cases. Only 1 patient required laparoscopic hysterectomy due to the appearance of the fibroid through the external cervical os.

\section{Discussion}

Percutaneous RF myolysis efficiency reduces both volume and symptoms of uterine fibroids. These results suggest transabdominal ultrasound-guided RF myolysis as a valid alternative treatment for symptomatic fibroids, 
Table 1. Menorrhagia assessed by score from 0 to 3, and dysmenorrhea and intermenstrual pain assessed by visual analogue scale (VAS) from 0 to 10 , before and after percutaneous RF myolysis.

\begin{tabular}{ccc}
\hline & Baseline & 6 months \\
\hline Total $(\mathrm{n}=17)$ & & \\
Menorrhagia & $2.29(0.92)$ & $1.13(0.89)^{\mathrm{a}}$ \\
Dysmenorrhea & $5.76(3.31)$ & $2.75(3.32)^{\mathrm{b}}$ \\
Intermenstrual pain & $3.00(3.43)$ & $1.38(2.56)^{\mathrm{c}}$ \\
\hline
\end{tabular}

Values are mean \pm SD. ${ }^{a} \mathrm{P}=0.005$ versus the baseline assessment. ${ }^{b} \mathrm{P}=0.004$ versus the baseline assessment. ${ }^{c} \mathrm{P}=0.02$ versus the baseline assessment.

Table 2. Fibroid volume and volume reduction after percutaneous RF myolysis in women with symptomatic uterine fibroids.

\begin{tabular}{ccccc}
\hline & \multicolumn{2}{c}{ Transvaginal ultrasound } & \multicolumn{2}{c}{ Magnetic resonance imaging } \\
\cline { 2 - 5 } & Baseline & 6 months & Baseline & 6 months \\
\hline Total $(\mathrm{n}=17)$ & & & & \\
Fibroid volume $\left(\mathrm{cm}^{3}\right)$ & $112.26(65.24)$ & $64.42(51.78)^{\mathrm{a}}$ & $118.71(74.88)$ & $94.57(75.32)^{\mathrm{b}}$ \\
Volume after reduction & 0 & $57.38 \%$ & 0 & $79.66 \%$ \\
\hline
\end{tabular}

Values are mean $\pm \mathrm{SD} .{ }^{\mathrm{a}} \mathrm{P}<0.001 .{ }^{\mathrm{b}} \mathrm{P}=0.019$

Table 3. Magnetic resonance imaging (MRI) and CEUS appearance of uterine fibroids before and after percutaneous RF myolysis.

\begin{tabular}{|c|c|c|}
\hline & Baseline $(\mathbf{n}=17)$ & 6 months $(n=14)$ \\
\hline \multicolumn{3}{|c|}{ Appearance of the MRI fibroid enhancement } \\
\hline Homogeneous & $15(88.24 \%)$ & $10(71.43 \%)$ \\
\hline Heterogeneous & 2 (11.77\%) & $4(28.57 \%)$ \\
\hline \multicolumn{3}{|l|}{ Fibroid necrosis (MRI) } \\
\hline$<50 \%$ & - & $1(7.14 \%)$ \\
\hline $50 \%-75 \%$ & - & $3(21.43 \%)$ \\
\hline$>75 \%$ & - & $10(71.43 \%)$ \\
\hline \multicolumn{3}{|l|}{ MRI T2-weighted signal } \\
\hline Hypointense and homogeneous & $4(23.53 \%)$ & $0(0.00 \%)$ \\
\hline Hypointense and heterogeneous & $9(52.94 \%)$ & $4(28.57 \%)$ \\
\hline Hyperintense and homogeneous & $1(5.88 \%)$ & $1(7.14 \%)$ \\
\hline \multirow[t]{2}{*}{ Hyperintense and heterogeneous } & $3(17.65 \%)$ & $9(64.29 \%)$ \\
\hline & Baseline $(\mathbf{n}=17)$ & 6 months $(n=15)$ \\
\hline \multicolumn{3}{|l|}{ CEUS appearance } \\
\hline Hypervascular & 17 (100.00\%) & $0(0.00 \%)$ \\
\hline Hypovascular & $0(0.00 \%)$ & $1(7.00 \%)$ \\
\hline Avascular & $0(0.00 \%)$ & $14(93.00 \%)$ \\
\hline
\end{tabular}

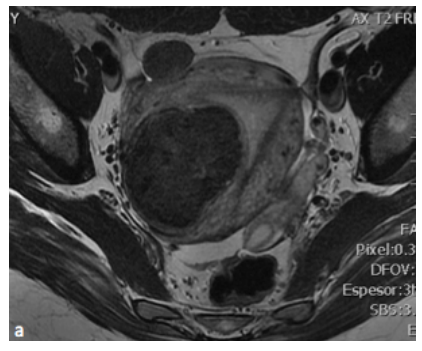

(a)

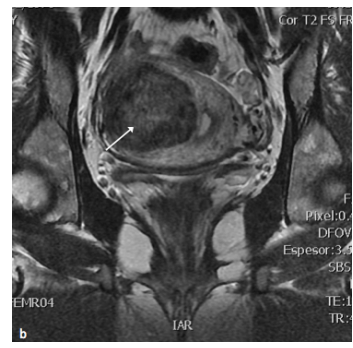

(b)

Figure 2. Transverse T2-weighted MRI image. Hypointensity of the fibroid prior to treatment (a); heterogeneous hyperintensity of the fibroid 6 months after treatment (arrow) (b). 


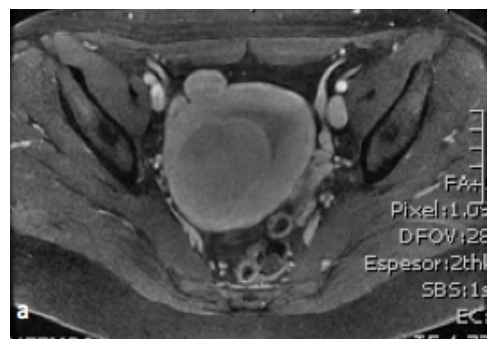

(a)

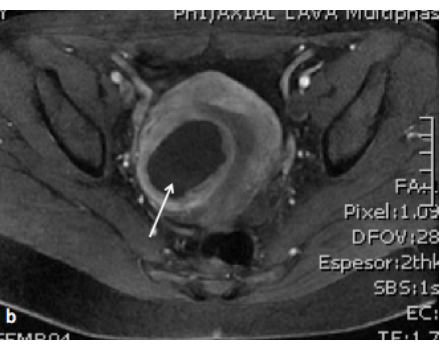

(b)

Figure 3. MR image in fat-suppressed T1-weighted sequence prior to treatment (a) and 6 months after treatment, which reveal an area of central necrosis (arrow) (b).

with encouraging efficacy and safety.

Current treatment of uterine fibroids is mainly based on surgical or radiological interventions, with limited medical treatment options [12]. Myolysis has been used in Europe since the late 1980s [1] [13]. Myolysis effectiveness using other energy sources such as monopolar coagulation, neodymium-doped yttrium aluminium garnet (Nd:YAG) laser and cryotherapy, and MRI-guided HIFU has been described [1] [10] [14]. However, RF ablation has been suggested as the method of choice because it is rapid, simple, predictable, safe and relatively inexpensive [1] [4] [15]. Initial studies showed the effectiveness of laparoscopic RF ablation by a mean decrease in uterine fibroid volume in the first 6 months of 77\% [16]. It was subsequently reported that the outcome of RF myolysis after $1-3$ years was encouraging in regard to reduction of persistent symptoms and improvement of the quality of life [15]. Although there is a lack of comparative studies with other conservative treatments, the improvement rate of symptom after myolysis compares favourably with that of myomectomy [17] or uterine embolization [18].

General anaesthesia is required in RF myolysis by laparoscopic approach [15] [16]. However, ultrasoundguided transabdominal RF myolysis requires a hospitalization time below 24 hours, allowing rapid recovery and early restoration of patient's normal activities [1]. In addition, RF myolysis can be more cost-effective than other methods, such as cryo myolysis or laparoscopic myolysis [1].

In the present study, mean ultrasound fibroid volume was $57.3 \%$ at six months after the procedure compared to baseline. This reduction is slightly inferior to that shown by Kim et al. [1] (68.6\%), Bergamini et al. [16] (77\%) and Ghezzi et al. [15] (68.8\%). The differences may be due to either a different resolution of ultrasound, initial tumor size or interobserver variability. The statistically significant reduction in dysmenorrhea, intermenstrual pain and bleeding six months after the intervention was similar to that reported by these authors [1] [15] [16].

According to the results showed by Kim et al. [1], Bergamini et al. [16], and Ghezzi et al. [15], major complications such as perforation or burn injury to bowel or bladder, sepsis or peritonitis are unusual and were not present during the procedure in our series.

Previous studies described dense fibrous adhesions between the fibroid and intestine after laparoscopic myolysis with Nd:YAG laser [13]. The presence of multiple post-puncture holes in the uterine serosa may increase the risk of postoperative adhesion formation [1]. Therefore, to reduce the risk of post-surgical adhesions, the fibroid should ideally be punctured only once to insert the needle with the electrode used in the ultrasoundguided procedure. In the present study, we performed an average of two ablations per patient with only one puncture. To minimize risk of adhesions, an attempt was made to use the same path in successive punctures, deepening or removing the needle without re-puncturing the fibroid and trying to cover most of it. The uterine attachment helps prevent damage to non-target structures and allow a safe access to posterolateral fibroids.

In any case, studies of laparoscopic and percutaneous puncture are comparable, safe and effective in terms of long-term results [5] [16]. A low rate of postoperative complications has been reported. In our study, these have been prompted by pain, which in all cases was controlled by analgesics.

Safety of radiofrequency myolysis in women with reproductive desire has not been established. Reported cases of full-term gestations that progressed without complication indicate that pregnancy is viable after myolysis [1]. Nevertheless, a large number of complications have been reported in relation to subsequent pregnancies, such as uterine rupture [1] [16]. This suggests that local tissue destruction without surgical repair may increase the risk of a suboptimal healing and uterine rupture during pregnancy after myolysis [1]. Due to insufficient 
information of the outcome of a pregnancy after myolysis, this technique should currently be considered as a treatment option in patients with reproductive desire in the context of a clinical trial [9]. Therefore, in the present study, only patients without reproductive desire were included.

Fibroids with greater cellular content or degenerative changes usually have high signal intensity on T2weighted sequences [19]. In our study, there is a predominance of treated fibroids with high signal intensity on T2-weighted sequences in MRI controls. This suggests progressive liquefaction, similar to that previously described by other studies [20]. This signal change is greater than the necrosis objectified in intravenous contrast perfusion. This suggests that the area of ablation exceeds that of necrosis, thus demonstrating efficiency. Compared with other ablation techniques such as HIFU or laser, percutaneous ablation is economical, quick, easy and repeatable. Furthermore, access via a single skin puncture reduces the use of general anaesthesia and complications arising therefrom. Suitable selection of patients is required regarding location, size, situation and accessibility to reduce damage to adjacent organs. Teamwork between gynaecologists and interventional radiologists is also important to perform a safe procedure.

The potential limitations of the present study are the limited number of patients, as well as the reduced followup time. Subsequent studies with a larger number of patients could be carried out in order to establish the safety and effectiveness of RF. In addition, in a unique study, the efficiency of RF could also be compared with that of other interventional techniques for uterine fibroids such as HIFU or laser. Finally, the safety of RF for women with uterine fibroids and pregnancy desire needs to be established.

Our study is comparable with previous articles and confirms uterine fibroid volume reduction and symptom improvement after percutaneous RF myolysis. Performing multiple thermalablations in the same procedure ensures greater volume treated with few complications. Larger series of patients and an adequate follow-up are necessary in order to optimize percutaneous thermal ablation in the management of symptomatic fibroids.

\section{References}

[1] Kim, C.H., Kim, S.R., Lee, H.A., Kim, S.H., Chae, H.D. and Kang, B.M. (2011) Transvaginal Ultrasound-Guided Radiofrequency Myolysis for Uterine Myomas. Human Reproduction, 26, 559-563. http://dx.doi.org/10.1093/humrep/deq366

[2] Bulun, S.E. (2013) Uterine Fibroids. New England Journal of Medicine, 369, 1344-1355. http://dx.doi.org/10.1056/NEJMra1209993

[3] Lumsden, M.A. (2002) Embolization versus Myomectomy versus Hysterectomy: Which Is Best, When? Human Reproduction, 17, 253-259. http://dx.doi.org/10.1093/humrep/17.2.253

[4] Jones, S., O’Donovan, P. and Toub, D. (2012) Radiofrequency Ablation for Treatment of Symptomatic Uterine Fibroids. Obstetrics and Gynecology International, 2012, Article ID: 194839. http://dx.doi.org/10.1155/2012/194839

[5] Carrafiello, G., Recaldini, C., Fontana, F., et al. (2010) Ultrasound-Guided Radiofrequency Thermal Ablation of Uterine Fibroids: Medium-Term Follow-Up. CardioVascular and Interventional Radiology, 33, 113-119. http://dx.doi.org/10.1007/s00270-009-9707-3

[6] Evans, P. and Brunsell, S. (2007) Uterine Fibroid Tumors: Diagnosis and Treatment. American Family Physician, 75, 1503-1508.

[7] Dueholm, M., Lundorf, E., Hansen, E.S., Ledertoug, S. and Olesen, F. (2002) Accuracy of Magnetic Resonance Imaging and Transvaginal Ultrasonography in the Diagnosis, Mapping, and Measurement of Uterine Myomas. American Journal of Obstetrics \& Gynecology, 186, 409-415. http://dx.doi.org/10.1067/mob.2002.121725

[8] Recaldini, C., Carrafiello, G., Laganà, D., et al. (2007) Percutaneous Sonographically Guided Radiofrequency Ablation of Medium-Sized Fibroids: Feasibility Study. American Journal of Roentgenology, 189, 1303-1306. http://dx.doi.org/10.2214/AJR.07.2184

[9] Kroon, B., Johnson, N., Chapman, M., Yazdani, A. and Hart, R. (2011) Australasian CREI Consensus Expert Panel on Trial Evidence (ACCEPT) Group. Fibroids in Infertility-Consensus Statement from ACCEPT (Australasian CREI Consensus Expert Panel on Trial Evidence). Australian and New Zealand Journal of Obstetrics and Gynaecology, 51, 289-295. http://dx.doi.org/10.1111/j.1479-828X.2011.01300.x

[10] Goldfarb, H.A. (2008) Myolysis Revisited. Journal of the Society of Laparoendoscopic Surgeons, 12, 426-430.

[11] Subramaniam, R., Vijayananthan, A., Omar, S., Nawawi, O. and Abdullah, B. (2010) Uterine Artery Embolisation for Symptomatic Fibroids: The University of Malaya Medical Centre Experience. The Biomedical Imaging and Intervention Journal, 6, e27. http://dx.doi.org/10.2349/biij.6.3.e27

[12] Donnez, J., Tomaszewski, J., Vázquez, F., et al. (2012) Ulipristal Acetate versus Leuprolide Acetate for Uterine Fi- 
broids. The New England Journal of Medicine, 366, 421-432. http://dx.doi.org/10.1056/NEJMoa1103180

[13] Donnez, J., Squifflet, J., Polet, R. and Nisolle, M. (2000) Laparoscopic Myolysis. Human Reproduction Update, 6, 609-613. http://dx.doi.org/10.1093/humupd/6.6.609

[14] Garza Leal, J.G., Hernandez Leon, I., Castillo Saenz, L. and Lee, B.B. (2011) Laparoscopic Ultrasound-Guided Radiofrequency Volumetric Thermal Ablation of Symptomatic Uterine Leiomyomas: Feasibility Study Using the Halt 2000 Ablation System. Journal of Minimally Invasive Gynecology, 18, 364-371. http://dx.doi.org/10.1016/j.jmig.2011.02.006

[15] Ghezzi, F., Cromi, A., Bergamini, V., Scarperi, S., Bolis, P. and Franchi, M. (2007) Midterm Outcome of Radiofrequency Thermal Ablation for Symptomatic Uterine Myomas. Surgical Endoscopy, 21, 2081-2085. http://dx.doi.org/10.1007/s00464-007-9307-8

[16] Bergamini, V., Ghezzi, F., Cromi, A., et al. (2005) Laparoscopic Radiofrequency Thermal Ablation: A New Approach to Symptomatic Uterine Myomas. American Journal of Obstetrics Gynecology, 192, 768-773. http://dx.doi.org/10.1016/j.ajog.2004.10.591

[17] Myers, E.R., Barber, M.D., Gustilo-Ashby, T., Couchman, G., Matchar, D.B. and McCrory, D.C. (2002) Management of Uterine Leiomyomata: What Do We Really Know? Obstetrics Gynecology, 100, 8-17. http://dx.doi.org/10.1016/S0029-7844(02)02019-7

[18] Spies, J.B., Bruno, J., Czeyda-Pommersheim, F., Magee, S.T., Ascher, S.A. and Jha, R.C. (2005) Long-Term Outcome of Uterine Artery Embolization of Leiomyomata. Obstetrics Gynecology, 106, 933-939. http://dx.doi.org/10.1097/01.AOG.0000182582.64088.84

[19] Yamashita, Y., Torashima, M., Takahashi, M., et al. (1993) Hyperintense Uterine Leiomyoma at T2-Weighted MR Imaging: Differentiation with Dynamic Enhanced MR Imaging and Clinical Implications. Radiology, 189, 721-725. http://dx.doi.org/10.1148/radiology.189.3.8234695

[20] Harman, M., Zeteroğlu, S., Arslan, H., Sengül, M. and Etlik, O. (2006) Predictive Value of Magnetic Resonance Imaging Signal and Contrast-Enhancement Characteristics on Post-Embolization Volume Reduction of Uterine Fibroids. Acta Radiologica, 47, 427-435. http://dx.doi.org/10.1080/02841850600557117

\section{Abbreviations}

Percutaneous radiofrequency (RF)

Magnetic resonance imaging (MRI)

Contrast enhanced ultrasound (CEUS)

Visual analogue scale (VAS)

High intesity focused ultrasound (HIFU)

Intrauterine devices (IUD)

Levonorgestrel (LNG)

Physiologic saline solution (PSS)

Neodymium-doped yttrium aluminium garnet (Nd:YAG) 
Scientific Research Publishing (SCIRP) is one of the largest Open Access journal publishers. It is currently publishing more than 200 open access, online, peer-reviewed journals covering a wide range of academic disciplines. SCIRP serves the worldwide academic communities and contributes to the progress and application of science with its publication.

Other selected journals from SCIRP are listed as below. Submit your manuscript to us via either submit@scirp.org or Online Submission Portal.
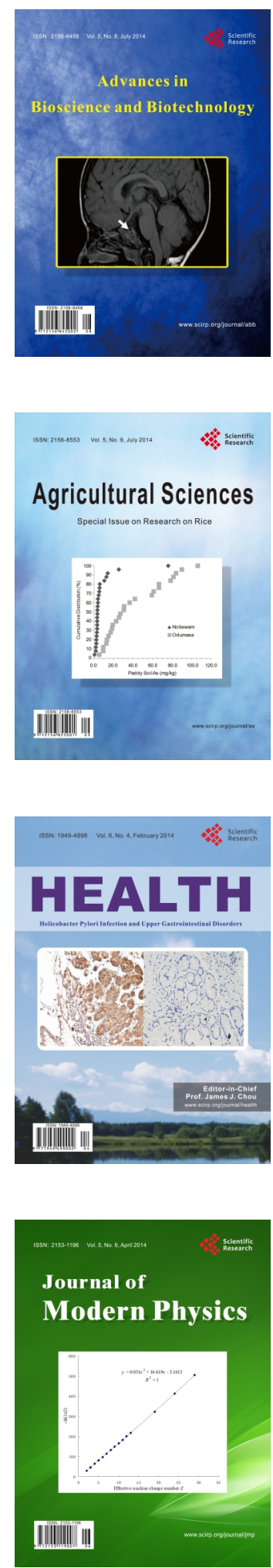
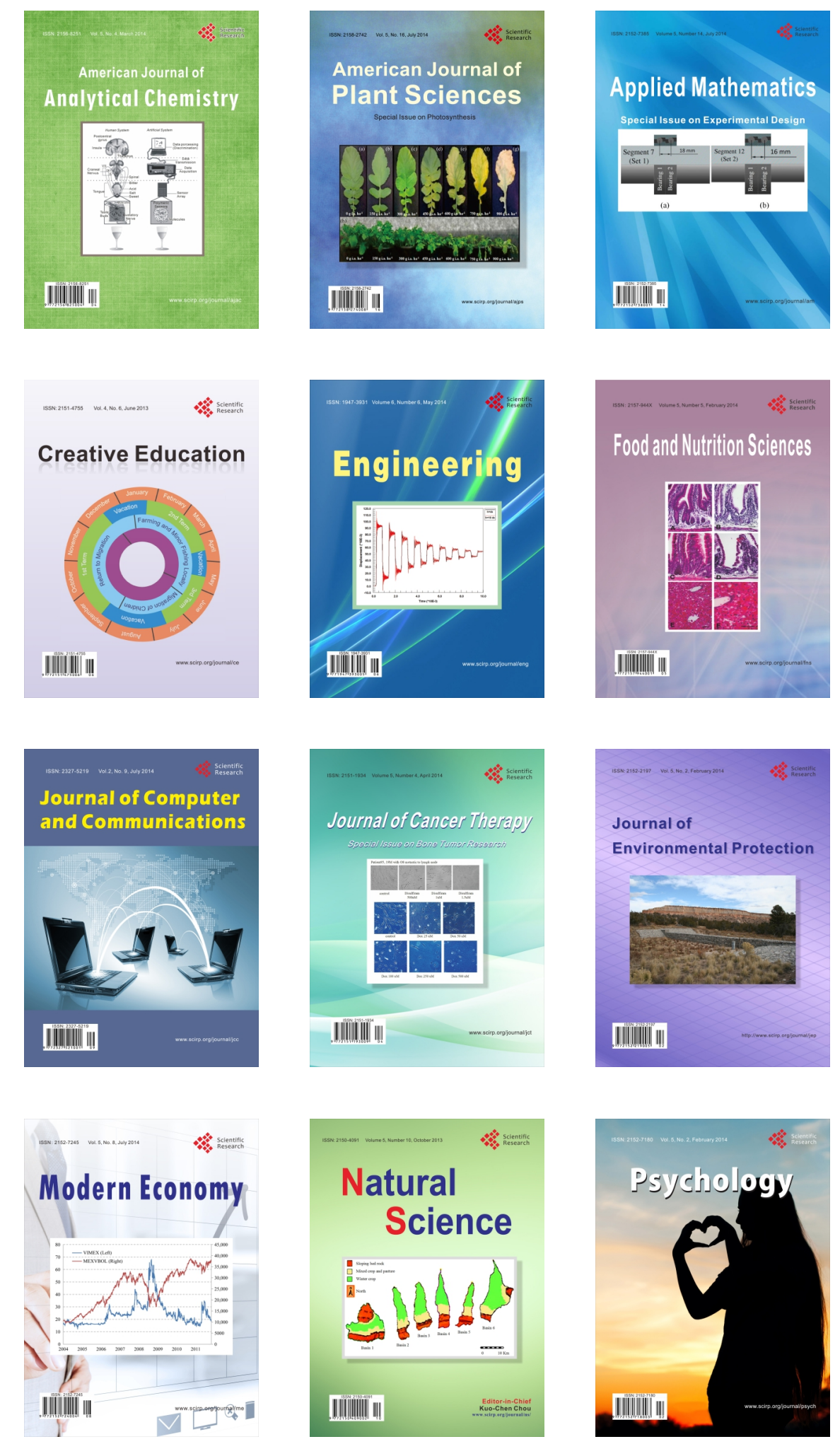